\title{
Pour une approche communicationnelle du travail d'organisation : changement organisationnel et gestion des événements indésirables
}

Isabelle Bazet, Alexia Jolivet et Anne Mayère

\section{(2) OpenEdition Journals}

Édition électronique

URL : http://journals.openedition.org/communicationorganisation/424

DOI : 10.4000/communicationorganisation.424

ISSN : $1775-3546$

Éditeur

Presses universitaires de Bordeaux

Édition imprimée

Date de publication : 1 juin 2008

Pagination : 30-39

ISBN : 978-2-86781-506-5

ISSN : $1168-5549$

Référence électronique

Isabelle Bazet, Alexia Jolivet et Anne Mayère, « Pour une approche communicationnelle du travail d'organisation : changement organisationnel et gestion des événements indésirables »,

Communication et organisation [En ligne], 33 | 2008, mis en ligne le 01 juin 2011, consulté le 30 avril

2019. URL : http://journals.openedition.org/communicationorganisation/424 ; DOI : 10.4000/

communicationorganisation. 424 
Dossier : Conduire le changement organisationnel?

\title{
Résumé
}

Les organisations contemporaines sont passées d'un travail d'optimisation des opérations, à un travail qui porte sur l'organisation elle-même, dans une logique d'amélioration continue. A partir de l'étude de la gestion des événements indésirables dans un établissement hospitalier, l'article interroge les formes de mobilisation des objets communicationnels associés et la pluralité des formes de communication et de production d'information, qui participent à l'acculturation au «système expert» qualité-risques, tout en constituant un espace de confrontation entre des approches différenciées.

\section{Mots-clés}

Réflexivité, gestion des risques, apprentissage organisationnel, objets communicationnels, confiance

\begin{abstract}
Organizations are currently focused on the continuous improvement of their process and organizational design. This paper, based on a research program concerning risk management in hospitals, questions the way communicational objects are used in such a change which deals with trust in risk management "expert system".
\end{abstract}

\section{Key-Words}

Reflexivity, risk management, organisational learning, communicational objects, trust

\section{Isabelle BAZET}

Isabelle Bazet est maître de conférences à l'IUT de Tarbes et chercheure au LERASS, équipe CHOCQ, Université Toulouse 3. Elle est aussi chercheure associée au CERTOP, Université Toulouse 2.

\section{Alexia JOLIVET}

Alexia Jolivet est doctorante au LERASS, équipe CHOCQ de l'Université Toulouse 3, et fait partie de l'école doctorale LLC des Universités Toulouse 2 et Toulouse 3.

\section{Anne MAYERE}

Anne Mayère est professeure à l'Université Toulouse 3 et à l'IUT A, responsable du M2R Information, Communication et Médiations Socio-Techniques. Elle est également chercheure au LERASS, équipe CHOCQ, Université Toulouse 3, et chercheure associée au CERTOP, Université Toulouse 2. 


\title{
Pour une approche communicationnelle du travail d'organisation : changement organisationnel et gestion des événements indésirables ${ }^{1}$
}

\author{
Isabelle Bazet, Alexia Jolivet, Anne Mayère
}

isabelle.bazet@iut-tarbes.fr alexia.jolivet@iut-tlse3.fr anne.mayere@iut-tlse3.fr

\section{Le travail d'organisation, régime ordinaire du changement}

Comme l'a bien identifié W. Orlikowski, le régime du changement est devenu le régime ordinaire des organisations contemporaines (Orlikowski, 1996). Dans leur recherche combinée de productivité accrue et d'adaptabilité, les organisations sont passées d'un travail d'optimisation des opérations, caractéristique de la période taylorienne et néo-taylorienne (Veltz, 2001), à un travail qui porte sur l'organisation elle-même, sur sa configuration, ses interfaces, dans une logique d'amélioration continue qui est aussi un changement de modèle d'organisation.

Pour mener à bien ce travail d'organisation (de Terssac, 2002), les différents personnels sont sollicités comme acteurs de la rationalisation. Ils sont amenés à participer à la mise en œuvre des reformulations organisationnelles et à leur finalisation. $\mathrm{La}$ rationalisation des organisations prend appui sur la diffusion de tout un ensemble de «dispositifs socio-techniques» tels que les certifications qualité ou la gestion des risques ; elle passe aussi par une acculturation à la «boite à outils» du travail organisationnel associé

\footnotetext{
${ }^{1}$ Cet article rend compte d'une partie des résultats d'un programme de recherche associant le CERTOP et le LERASS, programme PCCD/GEI qui bénéficie du soutien de la MiRe/DREES, et de la Région Midi-Pyrénées. Ont participé également à ce programme A. Roux et J. Simbille, Université Toulouse 3 IUT A, et M. Cattla, Université Toulouse 2. Ce programme est en lien avec la formation d'ingénieurs Informatique et Systèmes d'Information pour la Santé du CUFR JF Champollion, en partenariat avec les universités et écoles d'ingénieurs toulousaines.
} 
Dossier : Conduire le changement organisationnel?

(méthode de résolution de problèmes, arbre des causes et des conséquences, analyse de processus...).

En cela la question de la conduite du changement organisationnel ne peut être considérée comme celle de tout changement quel qu'il soit. Notre hypothèse d'interprétation, qui rejoint en cela d'autres travaux, est que ce changement s'inscrit dans une orientation globale, qui fait converger les entreprises et les organisations de services publics vers des caractéristiques communes (Bouillon, Durampart, 2006).

\section{Gestion des événements indésirables, objets communicationnels et confiance}

Pour étudier cette question, nous prendrons appui sur les résultats d'une recherche en cours concernant la gestion des événements indésirables (GEI) dans des établissements hospitaliers ${ }^{2}$. La logique de la GEI et les critères auxquels elle doit répondre sont définis dans diverses règlementations professionnelles, dans des lois et décrets, dans des vade-mecum établis par la tutelle ministérielle, par des experts ou des groupes de professionnels spécialisés. Par ailleurs, des logiciels spécialisés ont été développés, avec des fonctionnalités qui vont du formulaire de saisie des déclarations, à la base de données des événements et de l'état d'avancement des dossiers, jusqu'aux traitements statistiques et calcul d'indicateurs.

Cet ensemble de méthodes, d'instruments et d'outils, qui articulent très étroitement certification qualité et gestion des risques, peut être considéré comme un «système expert » au sens de Giddens ${ }^{3}$ (1994). Il est constitué notamment d'objets communicationnels, qui participent à

\footnotetext{
${ }^{2}$ Compte tenu de notre problématique nous avons retenu une méthodologie de type compréhensive. Premièrement, nous sommes parties des écrits, des énoncés, des traces pour comprendre les rationalités qui sous-tendent le travail ayant donné lieu à l'élaboration de la quantification et du repérage des événements, à la définition du circuit de déclaration et à leurs évolutions. Ensuite 39 entretiens semi - directifs ont été menés : auprès des membres de l'équipe de gestion des événements indésirables, auprès de membres du personnel, dans un premier temps en suivant le parcours du patient, puis au sein d'un même service et d'un service limitrophe. D'autre part, nous avons observé 18 réunions de gestion des événements indésirables, et 6 réunions de processus. Nous nous appuierons plus spécifiquement ici sur les investigations menées sur un des terrains d'observation.

3 «J'entends, par systèmes experts, des domaines techniques ou de savoir-faire professionnel concernant de vastes secteurs de notre environnement matériel et social. (...) Un système-expert délocalise de la même façon que les gages symboliques, en 'garantissant' nos attentes par rapport à un espace-temps lointain. » Giddens, 1994, p 36.
} 
mettre en forme les interactions et les productions d'information. En sont parties prenantes les dispositifs techniques mobilisés : intranet, logiciel dédié, courrier électronique, agenda partagé... ainsi que des principes et méthodes associés.

La mobilisation d'un tel système expert requiert qu'une confiance «suffisante» soit établie. Pour étudier ceci, nous reprenons la définition de Giddens, pour qui : "la confiance est un sentiment de sécurité justifié par la fiabilité d'une personne ou d'un système, et cette sécurité exprime une foi dans la probité ou l'amour d'autrui, ou dans la validité de principes abstraits (le savoir technologique)» (ibid, p. 41). Giddens distingue en cela la confiance-personne de la confiance-système, tout en montrant leur complémentarité. La première s'instaure dans des relations de face-à-face, alors que la seconde présuppose la foi dans des principes impersonnels.

En prenant appui sur une étude de cas approfondie, nous explorerons la synergie qui se met ou non en place entre communication médiatisée et communications de face à face, et participe à l'acculturation à cette nouvelle conception de l'organisation, de son évolution, et de ce qui fait le métier des professionnels concernés. Nous allons dans les points suivants étudier les tensions qui se font jour dans ce travail d'organisation entre désingularisation et resingularisation des événements, et entre dépersonnalisation et repersonnalisation des acteurs concernés.

\section{Le signalement : une communication médiatisée qui typifie l'événement}

La gestion des événements indésirables consiste à détecter et traiter des incidents qui sont survenus ou ont failli survenir, et qui sont facteurs de risque pour les patients ou pour le personnel. Tous les membres du personnel sont conviés à déclarer de tels événements. Chacun est ainsi réquisitionné pour assurer cette activité de «sentinelle».

La déclaration passe par le fait de remplir un formulaire, qui dans l'établissement étudié était sous format papier jusqu'à la mise en place de l'intranet en 2003, et qui est informatisé depuis lors. Ce formulaire est pour l'essentiel constitué de champs pré-définis, qui visent à caractériser le type d'événement et ce qui a pu en résulter, avec des cases à cocher entre des options pré-établies.

Même parmi ceux qui déclarent fréquemment, l'écrit préformaté de la déclaration est perçu comme insuffisant; en ramenant l'événement 


\section{Dossier : Conduire le changement organisationnel?}

singulier à des caractéristiques standards, il lui fait perdre de sa singularité. Pour décider de déclarer un événement, il faut avoir identifié ce qui a été jugé comme une situation a-normale, chargée émotionnellement puisque porteuse de risque effectif ou potentiel. Formaliser cet événement singulier dans une grille standard tend à le banaliser, à lui retirer en partie sa charge émotionnelle et la dimension atypique qui avait pu justifier le fait qu'il soit isolé comme événement indésirable, et que du temps et de l'énergie aient été temporairement dédiés à cette déclaration alors que tout, dans le rythme et la charge de travail, fait pression vers la poursuite de l'activité «ordinaire ».

\section{Mise en discussion et espace de parole}

Une fois la déclaration effectuée et reçue par la cellule qualité, cette dernière engage une investigation qui vise à mieux cerner l'événement et à en rechercher les causes organisationnelles. Dès lors, une communication de face-à-face fait suite à la communication médiatisée. Cette communication participe à la confiance dans le système expert, à la fois en ce qu'elle confirme l'effectivité de la prise en compte des déclarations, et en ce qu'elle s'inscrit dans une démarche précise, une démarche de professionnels de la qualité et de la gestion des risques, mobilisant des savoirs spécialisés. Elle permet de mieux exprimer la singularité de l'événement, mais elle est également très cadrée. A travers des instruments tels que les arbres des causes, les arbres des conséquences, le diagramme d'Ishikawa, etc., il s'agit de typifier la chaîne des actions qui sont intervenues de façon à identifier les actions significatives dans ce qui a pu contribuer à l'événement.

Cette démarche requiert donc la mise en œuvre d'une réflexivité au plan de l'organisation. Giddens identifie la réflexivité comme caractéristique de la modernité : «La réflexivité de la vie sociale moderne, c'est l'examen et la révision constantes des pratiques sociales, à la lumière des informations nouvelles concernant ces pratiques même, ce qui altère constitutivement leur caractère. » (1994, p 45). La réflexivité ici promue, à laquelle ce système expert tend à acculturer le personnel, est une réflexivité organisationnelle.

Une de ses caractéristiques-clé tient à ce qu'elle met volontairement de côté la question des personnes concernées tant au plan des «victimes» actuelles ou potentielles que des acteurs du processus en cause. Ces instruments et les principes associés requièrent en cela une mise à distance de l'émotion, du caractère situé et personnalisé de l'événement, pour en établir les faits saillants au plan organisationnel. 
Cette mise à distance ne va pas de soi, et même les membres de la cellule en charge de cette investigation font état de la charge associée à cette étape. La «boite à outils» des méthodes d'investigation est invoquée comme une aide dans cette distanciation, et dans la capacité à maintenir l'orientation du travail réflexif. En ce sens, elle agit comme un «bouclier», comme une «armure»; il s'agit de rationaliser le discours, de décharger la situation d'une partie de sa charge affective.

La communication de face-à-face ainsi mobilisée présente des caractéristiques ambivalentes. D'une part, elle ouvre un espace de parole dans lequel le personnel peut faire part de ses difficultés, de cette charge qui n'a pas de place dans l'activité et que les événements viennent souligner ou accentuer. D'autre part, le fait qu'une logique précise et des méthodes d'analyse soient mobilisées oblige à travailler l'événement, en s'écartant des schémas implicites, et des registres émotionnels spontanés.

La réflexivité portée par les démarches de qualité et gestion du risque est avant tout organisationnelle, ce qui passe par un travail de dépersonnalisation. Nous allons en explorer plus avant les dimensions.

\section{Dépersonnalisation - repersonnalisation autour des EI : une approche par l'organisation}

La dépersonnalisation qui est la règle dans la GEI porte à la fois sur le déclarant et sur le ou les acteurs concernés par les actions potentiellement inadéquates. Il y a tout d'abord anonymisation du déclarant. Plus précisément, le déclarant se nomme sur la fiche de déclaration de façon à faciliter la recherche d'informations complémentaires par la cellule. Cet anonymat, qui n'en est pas un d'emblée, met en jeu la confiance dans le système expert comme dans les individus qui travaillent à ses points d'accès. La cellule s'engage à assurer l'anonymisation des déclarations : les tentatives éventuelles de responsables hiérarchiques ou de collègues visant à savoir qui a déclaré sont éconduites. Cependant, les modalités de mise en œuvre de cet anonymat s'avèrent en partie insuffisantes pour le préserver strictement, notamment du fait de ce que requiert la phase d'investigation, et en raison du caractère situé des événements concernés ( $c f$. infra).

Le second volet de cette dépersonnalisation a trait aux acteurs potentiellement impliqués dans l'événement indésirable. Un principeclé de la plupart des méthodes de gestion du changement, et en particulier des démarches qualité et de gestion des risques, consiste à 


\section{Dossier : Conduire le changement organisationnel?}

mettre de côté les accusations relatives aux personnes pour interroger les défauts potentiels de l'organisation concernée. Selon ces démarches, et comme le relaient les méthodes d'investigation qui font partie de la «boite-à-outils» associée, tout individu travaillant dans une organisation est "présumé innocent», il est supposé qu'il souhaite réaliser un travail «de qualité »; ce sont des défaillances au plan du «design organisationnel», des processus, qui sont les causes premières des éventuels dysfonctionnements. La réflexivité organisationnelle requise représente en cela un changement important au regard des pratiques spontanées, qui font usuellement la part belle aux questions de personnes.

Ceux qui ont tendance à résister à ce travail de dépersonnalisation trouvent un argument dans la difficulté qu'il y a à préserver dans les faits l'anonymat des déclarants. En effet, lors de la phase de recherche des causes organisationnelles, les membres de la cellule qualité vont rencontrer des membres du personnel, dont souvent le ou la déclarant(e). L'anonymat de la déclaration en est relativement affaibli, d'autant que l'événement en question ne met souvent en présence qu'un nombre réduit de personnes, et qu'il aura souvent fait l'objet de discussions au sein du service.

Les membres du personnel qui adhèrent nettement au dispositif prennent de ce fait souvent les devants en annonçant leur déclaration à leurs collègues. Mais ce flou sur la préservation de l'anonymat participe à la réticence d'une autre partie du personnel, qui met plus particulièrement en avant la question de la délation. Cette question participe d'une appréhension, d'une crainte au regard du système expert, qui met en doute les principes même de ce travail d'organisation à l'échelle de l'institution.

\section{La crainte de la délation, une tension entre cohésion locale et efficacité globale}

Dans l'établissement étudié, les indicateurs de GEI permettent de considérer qu'il y a succès, au moins relatif, à travers le nombre de déclarations, et les types de déclarations identifiés. Or, les entretiens auprès de membres du personnel n'en font pas moins apparaître des ambiguïtés voire des contradictions.

Le mot capital présent dans les entretiens menés auprès du personnel est bien celui de «délation» (Jolivet, 2007, p. 52), qui se dégage ainsi comme une interpellation centrale vis-à-vis de laquelle chaque membre du personnel est conduit à se positionner. 
Dans cette ambiguïté qui se fait jour entre déclaration pour le bien du patient et crainte vis-à-vis des relations aux collègues et au collectif, intervient une tension entre la logique de délocalisation portée par les démarches qualité et de gestion des risques, et l'appartenance située, locale, au collectif. L'action de la cellule qualité-risques vise à démontrer la possibilité qu'il y aurait de combiner qualité du soin au patient et préservation des individus au travail, mais la difficulté que rencontre la cellule à totalement mettre à distance les questions de personnes ne facilite pas, pour une partie du personnel, le dépassement de cette crainte.

Il est ainsi possible d'identifier deux formes de représentation du dispositif. L'une envisage la situation comme celle d'un rapport inter-individuel, d'un individu portant un jugement sur les pratiques individuelles d'un autre, et renvoie à la question de la dénonciation. L'autre donne priorité au niveau organisationnel et valorise l'espace de dialogue ainsi ouvert sur les pratiques; prenant acte de la diversité des savoirs et des groupes professionnels, il s'agit d'en mettre en discussion tous les aspects, de mettre en lien les différents acteurs, pour améliorer la qualité et la sécurité de l'action collective. Cette seconde approche donne le primat à l'institution sur le groupe des pairs.

Parmi les membres du personnel qui ne déclarent pas, et en particulier parmi les plus réfractaires à la déclaration, une autre référence importante est celle du rapport à la hiérarchie. Selon ces personnes, ce serait au cadre que reviendraient le droit et le devoir de régler les dysfonctionnements. Il en serait ainsi en particulier dans la mesure où ce cadre connaît son personnel. Le raisonnement s'inscrit donc de façon orthogonale au regard du système expert de la gestion des risques, puisqu'il s'agit d'aborder le problème par les personnes.

On peut considérer que le personnel fait ici référence à un autre système expert, plus traditionnel, celui de l'organisation hiérarchique et de ses principes et méthodes de mise en œuvre. Il y a conflit de légitimité entre des systèmes experts différents. Le système expert «qualité risques » suppose un changement de paradigme, mais il reste inscrit dans une institution caractérisée par un pouvoir hiérarchique fort. La possibilité d'extraire les processus organisationnels de leurs «adhérences » aux personnes et aux actions individuelles n'est pas jugée crédible. Les membres du personnel concernés optent pour la référence au cadre organisationnel traditionnel, qui ne propose pas de dissocier fonctionnement organisationnel et rapports interpersonnels. 
Dossier : Conduire le changement organisationnel?

Lorsque le réseau de sociabilité de référence est essentiellement centré sur le service, le fait de déclarer suscite des réserves marquées en lien avec le risque perçu de désaffiliation au regard de ce collectif, auquel s'ajoute la crainte de réaction de la part de la hiérarchie. A contrario, le primat donné à l'institution et l'adhésion à la gestion des risques apparaissent d'autant plus aisés quand le réseau social est plus étendu, que les individus concernés savent ou pensent disposer d'options alternatives, peuvent mobiliser des réseaux qui dépassent leur service, leur groupe professionnel, voire leur institution. En cela la question relève bien de l'histoire des rapports aux mondes sociaux constitués.

\section{Conclusion}

Pour Y. Engeström (2001), les contradictions sont une dimension caractéristique de tout système d'activité ; elles sont le moteur du changement, d'une dynamique d'évolutions sans cesse renouvelées. K. Weick souligne, de façon complémentaire, l'importance des controverses, des espaces de débat, des confrontations de représentations, dans des organisations soumises à des risques constants et qui doivent faire preuve de résilience, tirer des enseignements de l'expérience pour faire face à des situations évolutives $(2002 ; 2006)$.

Le système expert qualité-risques, quand il ne se limite pas à constituer une chambre d'enregistrement de faits périphériques, peut constituer un cadre facilitateur tant pour l'acculturation à la réflexivité organisationnelle que pour la mise en débat d'approches différenciées. Il s'agit dès lors de prendre au sérieux les oppositions ou résistances à de tels dispositifs, en ce qu'elles en soulignent les limites, les impensés. Lorsque les oppositions s'ancrent dans des conceptions radicalement différentes de ce qui fonde l'activité et l'appartenance professionnelle, une communication managériale instrumentalisante, fondée sur des injonctions mettant en exergue la responsabilité de chacun, s'avère à la fois inefficace et éthiquement discutable. En revanche, la pluralité des objets communicationnels mobilisés dans de tels dispositifs - ou en articulation - avec eux peut participer à ce qui, selon K. Weick, favorise une élaboration collective du sens dans des situations marquées par une forte équivocité sociale des problèmes (Vidaillet, 2006). 
Pour une approche communicationnelle du travail d'organisation

\section{Bibliographie}

BAZET, Isabelle, MAYERE, Anne, JOLIVET, Alexia, ROUX, Angélique, CATTLA, Michel, SIMBILLE, Jocelyne. Conception et appropriation d'un dispositif de gestion d'événements indésirables Confiance, défiance et territoires de l'indésirable, rapport CERTOP LERASS pour la MiRe DREES, Décembre 2007, 139 p.

BOUILLON, Jean-Luc, DURAMPART, Michel. Entre autorégulation, formalismes et constructions symboliques : les paradoxes des nouvelles formes organisationnelles. In : Actes du colloque Pratiques et usages organisationnels des sciences et technologies de l'information et de la communication, Rennes, sept. 2006, p. 57-63.

ENGESTRÖM, Yrjö. Expansive learning at work: toward an activity theoritical reconceptualization. Journal of Education and Work, 2001, vol. $14, \mathrm{n}^{\circ} 1$, p. 133-156.

GIDDENS, Anthony. Les conséquences de la modernité. Paris : L'Harmattan, 1994, 192p.

JOLIVET, Alexia. Confiance et dispositifs de communication dans le cadre d'un système de déclaration d'événements indésirables. Mémoire de Master 2 Recherche. M2R ICMST. Toulouse : Université

Toulouse 3, Juillet 2007, 115 p.

ORLIKOWSKI, Wanda. Improvising organizational transformation over time: a situated change perspective. Information System Research, 1996, vol. 7, n 1, p. 63-91.

TERSSAC, Gilbert de. Le travail : une aventure collective. Toulouse : Octarès éditions, 2002, 310 p.

VELTZ, Pierre. Le nouveau monde industriel. Paris : Gallimard, 2000, $230 \mathrm{p}$.

VIDAILLET, Bénédicte. Le sens de l'action. Karl E. Weick: sociopsychologie de l'organisation. Paris : Vuibert, 2006, 183 p.

WEICK, Karl. The reduction of medical errors through mindful interdependence. In: ROSENTHAL, M.M., SUTCLIFFE, K.M., eds. Medical Error. What do we know ? What do we do? San Francisco: Jossey-Bass, 2002, p. 177-199.

WEICK, Karl, SUTCLIFFE, Kathleen. Mindfulness and the quality of organizational attention. Organization Science, July-August 2006, vol. $17, \mathrm{n}^{\circ} 4$, p. $514-524$. 